\title{
Differential and integral methods for three-dimensional inverse scattering problems with a non-local potential
}

\author{
A E Yagle \\ Communications and Signal Processing Laboratory, Department of Electrical Engineering \\ and Computer Science, The University of Michigan. Ann Arbor, MI 48109, USA
}

Received 7 April 1987, in final form 8 September 1987

\begin{abstract}
The inverse scattering problem for the three-dimensional Schrödinger equation with a non-local potential diagonal in the radius variable is considered. Generalised Gel'fand-Levitan and Marchenko integral equations are derived using Gram-Schmidt orthogonalisations of free-space wave solutions. A generalised miracle equation is then used to recover the non-local potential. This is the first unified presentation of both the generalised Gel'fand-Levitan and Marchenko equations for this problem. New differential fast algorithms for solving these integral equations and recovering the potential are presented. An application to the problem of linear least-squares estimation of a random field is noted.
\end{abstract}

\section{Introduction}

The inverse scattering problem for the three-dimensional Schrödinger equation with a time-independent, local, non-spherically symmetric potential has received considerable attention recently, due to the development of generalised Gel'fand-Levitan and Marchenko integral equations by Newton [1-3]. These integral equations are generalisations of similar equations derived for the one-dimensional inverse scattering problems. Alternative derivations have been given for the generalised Marchenko equation in [4-8] and for the generalised Gel'fand-Levitan equation in [8].

The same problem with a non-local potential diagonal in the radius variable was solved by Kay and Moses [9, 10] using a generalised Marchenko equation. We refer to their equation as a Marchenko equation, rather than a Gel'fand-Levitan equation, since the range of integration is infinite. A generalised Gel'fand-Levitan equation with a finite range of integration was given by Moses in [11]. A Gel'fand-Levitan equation for the one-dimensional problem with a non-local ('parity-dependent') potential was given in [12]. More recently, the approach used in [6] can also be applied to the non-local potential problem to derive a generalised Marchenko equation. However, there is no unified treatment of both generalised Gel'fand-Levitan and Marchenko equations for this problem.

The significance of the three-dimensional inverse scattering problem with a nonlocal potential is as follows. First, the scattering data for the unknown scattering potential (or the spectral data for the unknown operator) generally consist of the 
scattering amplitude, which has five degrees of freedom. This results in an overdetermined, ill-posed problem if a local potential with three degrees of freedom is to be reconstructed, as first pointed out by Moses [13]. However, the overdetermination problem does not arise if a non-local potential diagonal in the radius is to be reconstructed (see [11] and equation (2.6) below). This suggests that the inverse scattering problem for a non-local potential is mathematically the fundamental problem. Second, we show in $\$ 5$ that the problem of computing the linear leastsquares estimate of a three-dimensional random field on the surface of a sphere of noisy observations can be formulated as an inverse scattering problem with a nonlocal potential (in fact, this was the motivation for the present work). Finally, the behaviour of some distributed systems with varying non-zero response times can be modelled by a non-local scattering potential.

This paper presents a unified treatment of both the generalised Gel'fand-Levitan and Marchenko integral equations for the case of a non-local potential, and also for the special case of a local potential. These two integral equations look similar, but the forms of their solutions are in fact quite different. The integral equations are derived using the orthonormality of the solutions to the Schrödinger equation, as in [8-11] and [14]; however, here they are interpreted as performing Gram-Schmidt orthogonalisations of free-space wave solutions. This approach was used in [8] to derive the generalised Gel'fand-Levitan equation; here it is used to derive the generalised Marchenko equation as well. A generalised miracle equation [1], or fundamental identity [4], is then used to recover the non-local potential, as in [9-11].

The major contribution of this paper is the development of fast algorithms for solving these integral equations. These algorithms operate differentially, as opposed to an integral equation, and require much fewer computations than the solution of the integral equations by discretisation and matrix inversion would require. They do this by taking advantage of the inherent structure of an inverse scattering problem that exists due to time causality. Although the inverse scattering problem with a non-local potential is not causal, it does possess a structure that allows a causal interpretation. It should be noted that the inverse problem for a layered medium excited by a point harmonic source in the sinusoidal steady state has no causal element, and yet a differential fast algorithm for this problem that employs a causal structure has been developed [15].

The paper is organised as follows. Section 2 introduces the inverse scattering problem with a non-local potential. Section 3 derives the generalised Gel'fand-Levitan and Marchenko integral equations, and discusses their relationship. Section 4 derives the differential fast algorithms that constitute alternatives to solving the integral equations. Section 5 applies the results of the paper to the problem of computing the linear least-squares estimate of a random field.

\section{The inverse scattering problem with a non-local potential}

\subsection{The inverse scattering problem}

The following inverse scattering problem is considered. The wavefield $\hat{u}(x, k)$ satisfies the Schrödinger equation with a non-local potential

$$
\left(\Delta+k^{2}\right) \hat{u}(x, k)=\int_{S^{2}} V(x, e) \hat{u}(|x| e, k) \mathrm{d} e
$$


where $x \in R^{3}, e$ is a unit vector, $S^{2}$ is the unit sphere and the non-local scattering potential $V(x, e)$ is real-valued, smooth and has compact support. It is also assumed that $V(x, e)$ does not induce bound states; a sufficient condition for this is for $V(x, e)$ to be non-negative. Note that this is the non-local potential considered in [9-11]; it can be interpreted as an integral operator on functions of $R^{3}$ that is diagonal in the radius $|x|$.

Taking the inverse Fourier transform of the Schrödinger equation results in the plasma wave equation [4]

$$
\left(\Delta-\partial^{2} / \partial t^{2}\right) u(x, t)=\int_{S^{2}} V(x, e) u(|x| e, t) \mathrm{d} e
$$

which clarifies the meaning of the non-local potential $V(x, e)$. Let the potential be probed with an impulsive plane wave. At each point $x$, a scattered field is produced that depends not only on the wave field at $x$, but on the wave field at all points on the sphere of radius $|x|$. Also, if the incident field used to probe the medium has reached point $x$, it will cause scattering at all points on the sphere of radius $|x|$. This is clearly non-causal scattering; however, note that no scattering will take place inside the sphere of radius $|x|$. Therefore, $u(x, t)$ is zero for $t<-|x|$. Thus there is still a causal structure of sorts.

\subsection{Scattering solution}

Two different sets of boundary conditions are specified, resulting in two different solutions. The scattering solution $\hat{\psi}\left(x, k, e_{\mathrm{i}}\right)$ has the boundary condition

$$
\begin{gathered}
\hat{\psi}\left(x, k, e_{\mathrm{i}}\right)=\exp \left(-\mathrm{i} k e_{\mathrm{i}} \cdot x\right)+(\exp (-\mathrm{i} k|x|) / 4 \pi|x|) A\left(k, e_{\mathrm{s}}, e_{\mathrm{i}}\right)+\mathrm{O}\left(|x|^{-2}\right) \\
\text { as }|x| \rightarrow \infty
\end{gathered}
$$

where the scattering amplitude $A\left(k, e_{\mathrm{s}}, e_{\mathrm{i}}\right)$ is defined as

$$
A\left(k, e_{\mathrm{s}}, e_{\mathrm{i}}\right)=-\iint_{S^{2}} \exp \left(\mathrm{i} k e_{\mathrm{s}} \cdot y\right) V(y, e) \hat{\psi}\left(|y| e, k, e_{\mathrm{i}}\right) \mathrm{d} e \mathrm{~d} y
$$

and $e_{\mathrm{i}}$ and $e_{\mathrm{s}}$ are unit vectors. In the time domain, this corresponds to an incident impulsive plane wave in the direction $e_{i}$ being used to probe the potential and being scattered in all directions. The scattering amplitude specifies the far-field behaviour of the wave field, and constitutes the scattering data.

The scattered field $u\left(x, t, e_{\mathrm{i}}\right)$ is defined as the total field minus the incident field. More specifically, we have

$$
\hat{\psi}\left(x, k, e_{\mathrm{i}}\right)=\exp \left(-\mathrm{i} k e_{\mathrm{i}} \cdot x\right)+\int_{-|x|}^{\infty} u\left(x, t, e_{\mathrm{i}}\right) \exp (-\mathrm{i} k t) \mathrm{d} t
$$

where the limits of integration are determined by the support of $\psi\left(x, t, e_{\mathrm{i}}\right), u\left(x, t, e_{\mathrm{i}}\right)$ is caused by interactions of the incident field $\exp \left(-\mathrm{i} k e_{\mathrm{i}} \cdot x\right)$ with the scattering potential.

\subsection{Significance of non-local potential}

For the special case of a local potential, i.e., a potential $V(x, e)=V(x) \delta(x /|x|-e)$ for which the scattering at $x$ depends only on the wave field at $x$, it is known [1] that the 


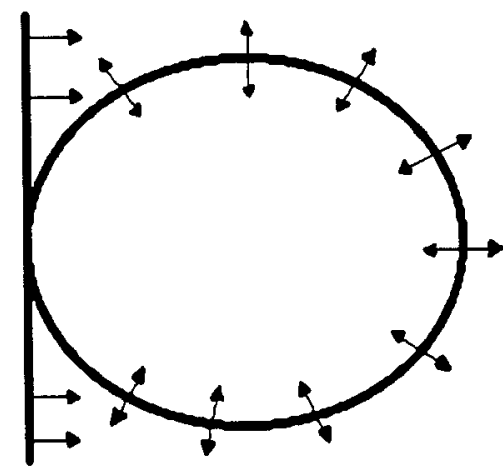

Figure 1. A probing plane wave passing through the non-local potential produces scattering over the surface of a sphere of radius $|x|=-t$.

inverse scattering problem of determining $V(x)$ from the scattering data $A\left(k, e_{\mathrm{s}}, e_{\mathrm{i}}\right)$ is overdetermined. This can be seen by noting that $A\left(k, e_{s}, e_{i}\right)$ has five degrees of freedom, while $V(x)$ has only three degrees of freedom. Thus most functions $A\left(k, e_{s}, e_{i}\right)$ will be inadmissible scattering amplitudes. This problem does not arise for the non-local potential $V(x, e)$, since this function has five degrees of freedom, matching the scattering data $A\left(k, e_{\mathrm{s}}, e_{\mathrm{i}}\right)$.

To see this explicitly, make the Born approximation of replacing the wavefield $\hat{\psi}\left(y, k, e_{i}\right)$ in (2.4) with the incident field $\exp \left(-i k e_{i} \cdot y\right)$, and let $y=|y| e_{y}$. Then (2.4) becomes

$A\left(k, e_{s}, e_{\mathrm{i}}\right)=-\iint_{s=} \int_{S=} \exp \left[\mathrm{i} k|y|\left(e_{\mathrm{s}} \cdot e_{y}-e_{\mathrm{i}} \cdot e\right)\right] V\left(|y| e_{y}, e\right)|y|^{2} \mathrm{~d} e \mathrm{~d} e_{y} \mathrm{~d}|y|$

which is a reversible transformation of $V(y, e)$ to $A\left(k, e_{s}, e_{i}\right)$. Thus the inadmissibility problem does not arise for the non-local potential.

Equation (2.6) can be interpreted physically using figure 1. As the incident plane wave reaches a point $x$, it causes scattering all around the sphere of radius $|x|$. In the Born approximation, this scattering propagates outward and becomes the scattering amplitude. By choosing $V(x, e)$ properly, any desired $A\left(k, e_{\mathrm{s}}, e_{\mathrm{i}}\right)$ can be produced. And since this can be done for each $e_{\mathrm{i}}$ (the contact with the sphere of radius $|x|$ occurs at $\left.x=-|x| e_{\mathrm{i}}\right)$, any reasonable $A\left(k, e_{\xi}, e_{\mathrm{i}}\right)$ can be produced by a choice of $V(x, e)$. Of course, $A\left(k, e_{s}, \mathrm{e}_{\mathrm{i}}\right)$ must be conjugate symmetric and reciprocal (see [1] and [4]).

\subsection{Regular solution}

The regular solution $\hat{\varphi}\left(x, k, e_{i}\right)$ is defined as being the solution to $(2.1)$ that is an entire analytic function of $k$ and is of exponential order $|x|$. This is the regular solution of Newton [3] the existence of which is proven in [3]; note that the regular solution defined in [1] is different and it has not been proven to exist. Using the Paley-Wiener theorem, the inverse Fourier transform $\varphi\left(x, t, e_{i}\right)=\mathscr{F}^{-1}\left\{\hat{\varphi}\left(x, k, e_{\mathrm{i}}\right)\right\}$ has support in $t$ on the interval $[-|x|,|x|]$. Thus it has the Povsner-Levitan representation [3] (compare with (2.5))

$$
\hat{\varphi}\left(x, k, e_{\mathrm{i}}\right)=\exp \left(-\mathrm{i} k e_{\mathrm{i}} \cdot x\right)+\int_{-|x|}^{|x|} m\left(x, t, e_{\mathrm{i}}\right) \exp (-\mathrm{i} k t) \mathrm{d} t
$$


so that $m\left(x, t, e_{\mathrm{j}}\right)$ is the scattered part of $\varphi\left(x, t, e_{\mathrm{i}}\right)$ in the sense that the regular solution would be merely the incident plane wave if the scattering potential were not present. Thus $m\left(x, t, e_{i}\right)$ for the regular solution is analogous to $u\left(x, t, e_{i}\right)$ for the scattering solution.

In the time domain the two solutions $\varphi\left(x, t, e_{i}\right)$ and $\psi\left(x, t, e_{i}\right)$ have different support, since the former has support in $t$ on the interval $[-|x|,|x|]$, while the latter has support in $t$ on the interval $[-|x|, \infty]$. Thus neither solution lies entirely inside the light cone; indeed the regular solution $\varphi\left(x, t, e_{\mathrm{i}}\right)$ lies entirely outside the light cone. For a local potential, $\psi\left(x, t, e_{\mathrm{i}}\right)$ has support on $[|x|, \infty]$, which does lie inside the light cone. Details on the relationship between the regular and scattering solutions for the one-dimensional problem are discussed in [16].

\subsection{Jost operator}

The two solutions are related by a Jost operator $J(k)$. This is an operator on the space $L^{2}\left(S^{2}\right)$ with kernel $J\left(k, e_{1}, e_{2}\right)$. Specifically,

$$
\begin{aligned}
& \hat{\varphi}\left(x, k, e_{\mathrm{i}}\right)=\int_{s^{2}} \hat{\psi}\left(x, k, e_{\mathrm{s}}\right) J\left(k, e_{\mathrm{s}}, e_{\mathrm{i}}\right) \mathrm{d} e_{\mathrm{s}} \\
& \hat{\psi}\left(x, k, e_{\mathrm{i}}\right)=\int_{s^{2}} \hat{\varphi}\left(x, k, e_{\mathrm{s}}\right) J^{-1}\left(k, e_{\mathrm{s}}, e_{i}\right) \mathrm{d} e_{s}
\end{aligned}
$$

where $J^{-1}$ is the inverse Jost operator. Both $J(k)$ and $J^{-1}(k)$ are analytic in the lower half-plane, which corresponds to causality in the time domain.

From [1], the Jost operator satisfies

$$
J(-k)=Q S(k) J(k) Q
$$

where $S(k)$ is the scattering operator with kernel

$$
S\left(k, e_{1}, e_{2}\right)=\delta\left(e_{1}-e_{2}\right)+(k / 2 \pi i) A\left(k, e_{1}, e_{2}\right)
$$

and $Q$ is the operator such that $Q A\left(k, e_{1}, e_{2}\right)=A\left(k,-e_{1}, e_{2}\right)$. Therefore the Jost operator can be computed from the scattering amplitude by solving an integral equation (see below).

\subsection{Orthonormality}

It is well known $[1,9,10]$ that in the absence of bound states the solutions $\left\{\hat{\psi}\left(x, k, e_{\mathrm{i}}\right)\right\}$ form a complete set. Thus they are orthonormal, in that

$$
(2 \pi)^{-3} \int_{0}^{\infty} \int_{S^{2}} \hat{\psi}(x, k, e) \hat{\psi}^{*}(y, k, e) k^{2} \mathrm{~d} e \mathrm{~d} k=\delta(x-y)
$$


It follows that the solutions $\left\{\varphi\left(x, k, e_{i}\right)\right\}$ are orthonormal with respect to the positive definite spectral function $\left(J^{\mathrm{H}} J\right)^{-1}$, i.e.,

$$
\begin{aligned}
(2 \pi)^{-3} \int_{0}^{\infty} \int_{s^{2}} \int_{s^{2}} \int_{s^{2}} \hat{\varphi}\left(x, k, e_{1}\right) J^{-1}\left(k, e_{1}, e_{3}\right) \\
\quad \times J^{-1}\left(k, e_{3}, e_{2}\right)^{*} \hat{\varphi}^{*}\left(y, k, e_{2}\right) k^{2} \mathrm{~d} e_{1} \mathrm{~d} e_{2} \mathrm{~d} e_{3} \mathrm{~d} k=\delta(x-y)
\end{aligned}
$$

as noted in [1] and [9-11].

\section{Integral equation solutions}

Two different integral equations for solving the inverse scattering problem are presented. The generalised Marchenko equation yields the scattering solution $\psi\left(x, t, e_{\mathrm{i}}\right)$, and the generalised Gel'fand-Levitan equation yields the regular solution $\varphi\left(x, t, e_{\mathrm{i}}\right)$. The non-local potential $V(x, e)$ is then reconstructed from either solution using a generalisation of the miracle equation [1]; for the non-local potential problem the miracle is no longer required.

The two integral equations are derived using an orthogonalisation procedure. This approach was used in [8] to derive the generalised Gel'fand-Levitan equation; we adapt it here to derive both integral equations, to show how they complement each other. Derivations of these equations were made in [3-8] for a local potential; a Marchenko equation for the non-local potential problem was derived in [10]. These derivations are intended to be illustrative rather than rigorous, since similar integral equations have been derived more rigorously elsewhere ([6, 9-11]).

\subsection{Generalised Marchenko equation}

In free space $V(x, e)=0$ the asymptotic form (2.3) will satisfy the Schrödinger equation (2.1), since (2.3) consists of a plane wave and an outgoing spherical wave. However, the orthonormality condition (2.11) must hold even where $V(x, e) \neq 0$. Therefore, the construction of the scattering solution $\hat{\psi}\left(x, k, e_{i}\right)$ can be regarded as a Gram-Schmidt orthogonalisation of the free-space solution (2.3), where the orthogonalisation starts at very large $|x|$ and proceeds inward in decreasing $|x|$. As the orthogonalisation progresses, the free-space solution (2.3) is replaced by the orthogonalised solution $\hat{\psi}\left(x, k, e_{i}\right)$, without changing the span of the solution for $|y|>|x|$. Each stage of the orthogonalisation constructs a $\hat{\psi}\left(x, k, e_{i}\right)$ that is orthogonal to span $\left[\hat{\psi}\left(y, k, e_{\mathrm{i}}\right),|y|>|x|\right]$ :

$$
\begin{aligned}
\hat{\psi}\left(x, k, e_{\mathrm{i}}\right) \perp & \operatorname{span}\left[\hat{\psi}\left(y, k, e_{\mathrm{i}}\right),|y|>|x|\right] \\
& =\operatorname{span}\left[\exp \left(-\mathrm{i} k e_{\mathrm{i}} \cdot y\right)+(\exp (-\mathrm{i} k|y|) / 4 \pi|y|) A\left(k, e_{\mathrm{s}}, e_{\mathrm{i}}\right),|y|>|x|\right] \\
& =\operatorname{span}\left[\exp \left(-\mathrm{i} k e_{\mathrm{i}} \cdot y\right)+\frac{1}{8 \pi^{2}} \int_{s^{2}} \mathrm{i} k A\left(k, e_{\mathrm{s}}, e_{\mathrm{i}}\right) \exp \left(-k e_{\mathrm{s}} \cdot y\right) \mathrm{d} e_{\mathrm{s}},|y|>|x|\right] .
\end{aligned}
$$


The latter equality is the Fourier transform of a relation given in [17].

Writing out the condition (3.1) gives, for $|y|>|x|$,

$$
\begin{aligned}
& \delta(x-y)=\iint_{S^{2}} \exp [-\mathrm{i} k e \cdot(x-y)] k^{2} \mathrm{~d} e \mathrm{~d} k \\
&+\frac{1}{8 \pi^{2}} \iint_{S^{2}} \int_{S^{2}} \mathrm{i} k A^{*}\left(k, e_{\mathrm{s}}, e\right) \exp \left[\mathrm{i} k\left(e_{\mathrm{s}} \cdot y-e \cdot x\right)\right] k^{2} \mathrm{~d} e_{\mathrm{s}} \mathrm{d} e \mathrm{~d} k \\
&+\iint_{S^{2}} u(x, k, e) \exp (\mathrm{i} k e \cdot y) k^{2} \mathrm{~d} e \mathrm{~d} k \\
&+\frac{1}{8 \pi^{2}} \iint_{S^{2}} \int_{S^{2}} u(x, k, e) \mathrm{i} k A^{*}\left(k, e_{\mathrm{s}}, e\right) \exp \left(\mathrm{i} k e_{\mathrm{s}} \cdot y\right) k^{2} \mathrm{~d} e_{\mathrm{s}} \mathrm{d} e \mathrm{~d} k .
\end{aligned}
$$

Cancelling the $\delta(x-y)$ term from both sides, defining

$$
G\left(t, e_{s}, e_{\mathrm{i}}\right)=\mathscr{F}^{-1}\left\{i k A\left(k, e_{\mathrm{s}}, e_{\mathrm{i}}\right)\right\}
$$

and performing the inverse Fourier transforms already present in (3.2) taking ke into $x$ and $k e_{\mathrm{s}}$ into $y$ results in

$$
\breve{u}(x, y)=\check{g}(x, y)+\int_{|x| \leqslant|z|} \check{u}(x, z) \check{g}(z, y) \mathrm{d} z \quad|y| \geqslant|x|
$$

where $\breve{g}(x, y)$ is the double inverse Radon transform of $G\left(t,-e_{\mathrm{s}}, e_{\mathrm{j}}\right.$ ) (see (3.3)). Equation (3.4) has the same form as (1.18) of [9]. A partial Radon transform [3] taking $y$ into $e_{i}$ yields the generalised Marchenko integral equation

$$
\begin{aligned}
u\left(x, t, e_{\mathrm{i}}\right)= & \int_{s^{2}} G\left(t-e_{\mathrm{s}} \cdot x, e_{\mathrm{s}}, e_{\mathrm{i}}\right) \mathrm{d} e_{\mathrm{s}} \\
& +\int_{-|.|}^{\infty} \int_{S^{2}} G\left(t+\tau,-e^{\prime}, e_{\mathrm{i}}\right) u\left(x, \tau, e^{\prime}\right) \mathrm{d} e^{\prime} \mathrm{d} \tau
\end{aligned}
$$

which holds for $|t|>|x|$. Note that this has the same form as the Marchenko equation of [6].

The scattered field $u\left(x, t, e_{\mathrm{i}}\right)$ in the time domain is thus recovered from the scattering amplitude $A\left(k, e_{s}, e_{\mathrm{i}}\right)$ by inverse Fourier transforming the latter, as in (3.3), and then solving the integral equation (3.5). Note the coupling in $e^{\prime}$ in the last term of (3.5). Note also that the range of integration is infinite, reflecting the support of $\psi(x, t$, $\left.e_{i}\right)$; hence this is a Marchenko equation.

\subsection{Generalised Gel'fand-Levitan equation}

To construct the generalised Gel'fand-Levitan equation, the orthonormality (2.12) of the regular solutions $\hat{\varphi}\left(x, k, e_{\mathrm{i}}\right)$ with respect to the weighting $\left(J^{\mathrm{H}} J\right)^{-1}$ is used. Also, the Gram-Schmidt orthonormalisation takes place in increasing $|x|$, and the free-space wavefunctions being orthogonalised are the plane waves $\exp \left(-\mathrm{i} k e_{i} \cdot x\right)$. Equation (3.1) is replaced with

$\hat{\varphi}\left(x, k, e_{\mathrm{i}}\right) \perp \operatorname{span}\left[\hat{\varphi}\left(y, k, e_{\mathrm{i}}\right),|y|<|x|\right]=\operatorname{span}\left[\exp \left(-\mathrm{i} k e_{\mathrm{i}} \cdot y\right),|y|<|x|\right]$ 
where the orthogonality includes the weight $\left(J^{\mathrm{H} J}\right)^{-1}$. Writing out (3.6) gives, for $|y|<|x|$

$$
\begin{aligned}
\delta(x-y)=\iint_{s^{2}} \int_{s^{2}} \exp \left(-\mathrm{i} k e_{1} \cdot x\right)\left(J^{\mathrm{H} J}\right)^{-1}\left(k, e_{1}, e_{2}\right) \exp \left(\mathrm{i} k e_{2} \cdot y\right) k^{2} \mathrm{~d} e_{1} \mathrm{~d} e_{2} \mathrm{~d} k \\
+\iint_{s^{2}} \int_{s^{2}} \hat{m}\left(x, k, e_{1}\right)\left(J^{\mathrm{H} J} J\right)^{-1}\left(k, e_{1}, e_{2}\right) \exp \left(\mathrm{i} k e_{2} \cdot y\right) k^{2} \mathrm{~d} e_{1} \mathrm{~d} e_{2} \mathrm{~d} k
\end{aligned}
$$

which can be rewritten as (compare with (3.4))

$$
\check{H}(x, y)=\check{m}(x, y)+\int_{|z| \leqslant|x|} \check{m}(x, z) \check{H}(z, y) \mathrm{d} z \quad|y| \leqslant|x|
$$

where

$$
\begin{array}{r}
\check{H}(x, y)=(2 \pi)^{-3} \int_{0}^{\infty} \int_{S^{2}} \int_{S^{2}}\left(\left(J^{\mathrm{H}} J\right)^{-1}-I\right)\left(k, e_{1}, e_{2}\right) \\
\quad \times \exp \left[-\mathrm{i} k\left(e_{1} \cdot x-e_{2} \cdot y\right)\right] k^{2} \mathrm{~d} e_{1} \mathrm{~d} e_{2} \mathrm{~d} k,
\end{array}
$$

$I$ represents $\delta\left(e_{1}-e_{2}\right)$ and $\check{m}(x, y)$ is the partial inverse Radon transform [3] of $M(x, t$, $e_{\mathrm{i}}$ ). Equation (3.8) has the same form as (2.7) of [11]. Defining

$$
M\left(t, e_{1}, e_{2}\right)=\mathscr{F}^{-1}\left\{\left(J^{\mathrm{H} J}\right)^{-1}\left(k, e_{1}, e_{2}\right)\right\}
$$

and taking the partial Radon transform [3] of (3.8) results in the generalised Gel'fand-Levitan equation:

$$
\begin{aligned}
m\left(x, t, e_{\mathrm{i}}\right)= & \int_{s^{2}} M\left(t+e_{\mathrm{i}} \cdot x, e_{s}, e_{\mathrm{i}}\right) \mathrm{d} e_{\mathrm{s}} \\
& -\int_{s^{2}} \int_{-|x|}^{|x|} m\left(x, \tau, e_{s}\right) M\left(t+\tau, e_{s}, e_{\mathrm{i}}\right) \mathrm{d} \tau \mathrm{d} e_{s}
\end{aligned}
$$

which holds for $|t|<|x|$. Note that this is the same equation as in [3], but it applies for a non-local potential.

The regular solution is thus recovered from the inverse Jost operator $J^{-1}(k)$ by inverse Fourier transforming $\left(J^{\mathrm{H} J}\right)^{-1}$, as in (3.10), and then solving the integral equation (3.11). Note the finite range of integration in (3.11), reflecting the support of $\varphi\left(x, t, e_{i}\right)$. This makes the Gel'fand-Levitan equation (3.11) easier to solve than the Marchenko equation (3.5), since the latter has an infinite range of integration. However, it is necessary to construct the inverse Jost operator from the scattering data using (2.10). This amounts to solving the integral equation $[1,8]$

$L\left(t, e_{s}, e_{\mathrm{i}}\right)=G\left(t,-e_{s}, e_{\mathrm{i}}\right)+\int_{11}^{\infty} \int_{S^{2}} L\left(\tau,-e_{s}, e^{\prime}\right) G\left(t+\tau, e^{\prime}, e_{\mathrm{i}}\right) \mathrm{d} e^{\prime} \mathrm{d} \tau$

for $L\left(t, e_{\mathrm{s}}, e_{\mathrm{i}}\right)$, where $G\left(t, e_{1}, e_{2}\right)$ is defined in (3.4). Then $J^{-1}\left(k, e_{1}, e_{2}\right)$ is reconstructed using

$$
J^{-1}\left(k, e_{1}, e_{2}\right)=1+\int_{0}^{\infty} L\left(t, e_{1}, e_{2}\right) \exp (-\mathrm{i} k t) \mathrm{d} t
$$




\subsection{Comparison of integral equations}

The integral equations (3.5) and (3.11) look similar, but they are in fact quite different. The Marchenko equation (3.5) requires as data the scattering amplitude, has an infinite range of integration and yields the scattered part $u\left(x, t, e_{i}\right)$ of the scattering solution. The Gel'fand-Levitan equation (3.11) requires as data the Jost operator, has a finite range of integration and yields the scattered part $m\left(x, t, e_{\mathrm{i}}\right)$ of the regular solution.

The similarities between the two integral equations encompass more than their forms. It has been shown that both can be interpreted as performing orthogonalisations of free-space wave solutions, with the orthogonalisations proceeding in different directions. The transformations (3.4) and (3.10) made on the data are identical, and both integral equations admit fast algorithm solutions (see $\$ 4$ ). Burridge [16] discusses the relationships between these equations in detail in the one-dimensional case.

In the special case of a local potential, the similarity is even more striking. In this case, the lower limit on the range of integration in (3.5) is changed from $-|x|$ to $|x|$, since for a local potential the scattered field will be zero for $t<e_{\mathrm{i}} \cdot x<|x|$. In this case the ranges of integrations in (3.5) and (3.11) complement each other, intersecting on the border $t=|x|$ of the light cone. It should be noted here that the solution to the integral equations in [9] and [10] is not the scattering solution $\psi\left(x, t, e_{i}\right)$, and this makes recovery of the scattering potential more difficult (see below).

It is not surprising that the integral equations for problems with non-local potentials are so similar to those for problems with local potentials. In [6] a Green's function argument is used to show that the Marchenko equation should have the same form for any inverse scattering problem with a smooth, compact potential, since it is merely an expression of Green's theorem. Note that in the general Marchenko equation of [6] there is a $u(x,-t,-e)$ term that is eliminated by causality; for the non-local potential problem considered here this term is still eliminated, since the scattered field is zero for $t<-|x|$.

\subsection{Recovery of scattering potential}

The potential $V(x, e)$ is recovered from the integral equation solutions as follows. The procedure for the scattering solution is simpler than the procedure for the regular solution, since the former has a simple physical interpretation. The scattering solution is considered first.

Taking the inverse Fourier transform of the definition (2.5) of the scattered field yields

$$
\psi\left(x, t, e_{\mathrm{i}}\right)=\delta\left(t-e_{\mathrm{i}} \cdot x\right)+u\left(x, t, e_{i}\right) 1(t+|x|)
$$

where 1() is the unit step or Heaviside function whose presence in (3.14) simply affirms that $u\left(x, t, e_{i}\right)$ is zero for $t<-|x|$. Inserting (3.14) into the plasma wave equation (2.2) results in

$$
\int_{S=2} V(x, e) \delta\left(t-e_{\mathrm{i}} \cdot e|x|\right) \mathrm{d} e=2 e_{x} \cdot \nabla u\left(x, t, e_{\mathrm{i}}\right) \delta(t+|x|)
$$

which becomes

$$
V\left(x,-e_{\mathrm{i}}\right)=2 e_{x} \cdot \nabla u\left(x, t=-|x|, e_{\mathrm{i}}\right)
$$


which is a generalisation of the miracle equation of [1] or the fundamental identity of [4]. Equation (3.16) can be used to reconstruct the potential $V(x, e)$ from the scattered field $u\left(x, t, e_{i}\right)$.

Equation (3.16) can be physically interpreted as follows. At the instant $t=-|x|$, the probing impulsive plane wave $\delta\left(t-e_{i} \cdot x\right)$ is tangent to the sphere of radius $|x|$ (see figure 1). Since the potential is non-local, scattering is induced over the surface of this sphere. The jump in the gradient of the scattered field at any point $x$ on the sphere of radius $|x|$ results from $V\left(x,-e_{\mathrm{i}}\right)$, since it is caused by the impulsive plane wave intersecting the sphere at point $-|x| e_{j}$. Furthermore, the scattered field will propagate inward, but no faster than the impulsive plane wave. Hence the field inside the sphere is zero, and the jump in the gradient of the scattered field is simply its value. This allows the entire scattering potential $V(y, e)$ to be computed for all points $y$ on the sphere of radius $|x|$.

The regular solution $\varphi\left(x, t, e_{\mathrm{i}}\right)$ can also be used to reconstruct $V(x, e)$, but here the procedure is more complicated. Since [1] $\varphi(x, t, e)=\varphi(x,-t,-e)$, the regular solution is a Radon transform of some function $\varphi(x, y)$. An inverse Fourier transform of the Povsner-Levitan relation (2.7) yields

$$
\check{\varphi}(x, y)=\delta(x-y)+\check{m}(x, y) 1(|x|-|y|)
$$

where the unit step affirms that $\check{m}(x, y)$ is zero unless $|y|<|x|$. Another way to derive (3.17) is to note that since $\varphi\left(x, t, e_{\mathrm{i}}\right)$ is zero if $|t|>|x|$, its inverse Radon transform $\dot{\varphi}(x, y)$ is zero if $|y|>|x|$. An inverse Radon transform of the plasma wave equation (2.2) results in

$$
\left(\Delta_{x}-\Delta_{y}\right) \dot{\varphi}(x, y)=\int_{S^{2}} V(x, e) \dot{\varphi}(|x| e, y) \mathrm{d} e .
$$

Equation (3.18) appears in [9]; its version for a local potential appeared in [3] and [18]. Inserting (3.17) into (3.18) gives [11]

$$
V(x, e)=-\frac{2}{|x|^{2}} \frac{\mathrm{d}}{\mathrm{d}|x|}|x|^{2} \check{m}(x,|x| e)
$$

which can be used to reconstruct $V(x, e)$ from $m\left(x, t, e_{\mathrm{i}}\right)$ or its inverse Radon transform $\check{m}(x, y)$.

Note that since the regular solution $\varphi\left(x, t, e_{\mathrm{i}}\right)$ does not have an obvious physical interpretation as does the scattering solution $\psi\left(x, t, e_{i}\right)$, equation (3.19) is more complicated than (3.16). There is no simple physical principle like that explained above for the scattering solution, and so the Radon transform must be employed. Equations similar to (3.19) appear in [9] and [10] in the context of inverse scattering problems and in [19] and [20] in the context of random fields estimation (see $\$ 5$ below).

For the special case of a local potential, equation (3.16) reduces to the miracle equation of [1]:

$$
V(x)=-2 e_{\mathrm{i}} \cdot \nabla u\left(x, t=e_{\mathrm{i}} \cdot x, e_{\mathrm{i}}\right),
$$

since (3.16) is then singular at $e_{\mathrm{i}}=-e_{x}$. Note that the right-hand side of (3.20) must be independent of the direction of incidence $e_{i}$. This is the 'miracle', and it imposes a constraint on possible $\hat{\psi}\left(x, k, e_{i}\right)$ and hence on possible scattering amplitudes $A\left(k, e_{s}, e_{i}\right)$. For the non-local potential, no 'miracle' is necessary. This suggests that 
the non-local potential inverse scattering problem is in some sense the fundamental problem.

The use of the Radon transform requires some discussion. For the regular solution it is true that $\varphi(x, t, e)=\varphi(x,-t,-e)$, so that its inverse Radon transform $\varphi(x, y)$ is well defined. However, for the scattering solution we have $\psi(x, t, e) \neq \psi(x,-t,-e)$, so that while the inverse Radon transform may be taken, it is not possible to Radon transform $\breve{u}(x, y)$ back to $u(x, t, e)$. Fortunately, this is not necessary. However, note that in [9] and [10] it is necessary to operate in the Radon transform domain and reconstruct $V(x, e)$ using (3.19), since the integral equations in [9] and [10] are similar to $(3.4)$.

The major disadvantage of solving the inverse scattering problem using either of the above integral equations is the tremendous amount of computation required to discretise and solve them. In the next section fast algorithms are developed that require much less computation.

\section{Differential fast algorithm solutions}

Two fast algorithms for solving the inverse scattering problem are derived. One reconstructs the scattered field $u\left(x, t, e_{\mathrm{i}}\right)$ recursively in decreasing $|x|$, recovering the potential $V(x, e)$ as it proceeds. The other reconstructs the Radon transform $\breve{m}(x, y)$ of the scattered part of the regular solution in increasing $|x|$, also recovering the potential as it proceeds. The two algorithms operate differentially, as opposed to integral equations, and they require much less computation than solving the respective integral equations; the savings occur due to structure that manifests itself as the Hankel kernels of the integral equations, but is actually due to various types of causality. The algorithms can be considered to be generalised Krein-Levinson and fast Cholesky algorithms [21], due to their recursion patterns, the solutions they reconstruct and the presence of an inner product computation in the second algorithm.

\subsection{Generalised fast Cholesky algorithm}

This algorithm recursively reconstructs the scattered field $u\left(x, t, e_{i}\right)$ and potential $V(x, e)$ in decreasing $|x|$. Unlike the generalised Marchenko integral equation, which employs as data the far-field scattering amplitude $A\left(k, e_{s}, e_{\mathrm{i}}\right)$, this algorithm is initialised using near-field data. Specifically, the datum required is the back-scattered field $u\left(x, t, e_{\mathrm{i}}\right)$ measured over the surface of a sphere outside the support of $V(x, e)$. Note that for many problems (e.g. inverse seismic problems) data are taken in the near field. The plane-wave reflection response for all angles of incidence $e_{\mathrm{i}}$ is a situation common in tomographic problems.

The algorithm is derived as follows. The Laplacian operator $\Delta$ can be written as

$$
\Delta=\frac{\partial^{2}}{\partial x^{2}}+\frac{2}{x} \frac{\partial}{\partial x}+\Delta^{\mathrm{T}}
$$

where

$$
\Delta^{\mathrm{T}}=\frac{1}{x^{2} \sin \theta} \frac{\partial}{\partial \theta}\left(\sin \theta \frac{\partial}{\partial \theta}\right)+\frac{1}{x^{2} \sin ^{2} \varphi} \frac{\partial^{2}}{\partial \varphi^{2}}
$$


is the transverse radial Laplacian operator in spherical coordinates. Here and in the following we use $x$ to represent both position $x$ and its magnitude $|x|$; the choice will be obvious from context.

The plasma wave equation (2.2) can then be written as

$$
\left[\left(\frac{\partial^{2}}{\partial x^{2}}+\frac{2}{x} \frac{\partial}{\partial x}\right)-\frac{\partial^{2}}{\partial t^{2}}\right] u\left(x, t, e_{\mathrm{i}}\right)=U\left(x, t, e_{\mathrm{i}}\right)
$$

where

$$
U\left(x, t, e_{\mathrm{i}}\right)=-\Delta^{\mathrm{T}} u\left(x, t, e_{\mathrm{i}}\right)+\int_{S^{2}} V(x, e) u\left(|x| e, t, e_{\mathrm{i}}\right) \mathrm{d} e
$$

is an auxiliary quantity.

Equation (4.3) in turn can be written as the coupled system of first-order equations

$$
\begin{aligned}
& \left(\frac{\partial}{\partial x}-\frac{\partial}{\partial t}\right)|x| u\left(x, t, e_{\mathrm{i}}\right)=|x| Q\left(x, t, e_{i}\right) \\
& \left(\frac{\partial}{\partial x}+\frac{\partial}{\partial t}\right)|x| Q\left(x, t, e_{\mathrm{i}}\right)=|x| U\left(x, t, e_{\mathrm{i}}\right)
\end{aligned}
$$

where $Q\left(x, t, e_{\mathrm{i}}\right)$ is another auxiliary quantity defined in (4.5a). From equation (3.16) we also have

$$
V\left(x,-e_{\mathrm{i}}\right)=2 Q\left(x, t=-|x|, e_{\mathrm{i}}\right)
$$

where we have used the fact that only the gradient of the scattered field jumps at $t=-|x|$; the field itself is zero since it is zero for $t \leqslant-|x|$.

Equations (4.4)-(4.6) can be propagated recursively in decreasing $|x|$ for $t \geqslant-|x|$. The algorithm is initialised on the surface of a sphere which lies outside the support of $V(x, e)$. For each $e_{\mathrm{i}}$, equations (4.4)-(4.6) are propagated in decreasing $|x|$ as follows. Suppose the algorithm has proceeded to $\left|x_{0}\right|+\Delta$, so that all quantities are known on the surface of a sphere of this radius, for all $e_{\mathrm{i}}$. Then (4.5) are used to compute $u\left(x, t, e_{\mathrm{i}}\right)$ and $Q\left(x, t, e_{\mathrm{i}}\right)$ on the surface of the sphere of radius $\left|x_{i}\right|$, for all $e_{\mathrm{i}}$. Next, (4.6) is used to compute $V(x, e)$ on the surface of this sphere for all $e$. Finally, (4.4) is used to compute $U\left(x, t, e_{i}\right)$ on the surface of this sphere (note that the transverse Laplacian $\Delta^{\mathrm{T}}$ only requires values on the surface of the sphere). At this point all quantities are known on the surface of the sphere of radius $\left|x_{0}\right|$, and the recursion is complete.

The recursion patterns for this algorithm are illustrated in figure 2 . Note that by causality all quantities are zero for $t \leqslant-|x|$, so no information is lost in the unshaded regions. Note also that since this algorithm is propagated in decreasing $|x|$, the recursions work differently from those in [7] and [8]. However, the required value $V\left(x,-e_{\mathrm{i}}\right)=2 Q\left(x, t=-|x|, e_{\mathrm{i}}\right)$ is still computed automatically; this is not the case with the next algorithm.

This algorithm is termed a generalised fast Cholesky algorithm since it resembles the one-dimensional fast Cholesky or layer stripping algorithm [21] in the following ways. First, the quantities being propagated are wave-like, in that they propagate and are causal. Second, the scattering parameter (potential or reflection coefficient) is computed automatically as the algorithm runs. Finally, the scattering data are used to initialise the algorithm, and can be discarded once it starts. 


\subsection{Generalised Krein-Levinson algorithm}

This algorithm recursively reconstructs the inverse Radon transform of the scattered part of the regular solution $\check{m}(x, y)$, and the potential $V(x, e)$, in increasing $|x|$. Unlike the previous algorithm, it operates in the inverse Radon transform domain; this is necessary since (3.19) recovers the potential from $\breve{m}(x, y)$ instead of $m\left(x, t, e_{i}\right)$. This is permissible since the regular solution, unlike the scattering solution, is a Radon transform in that $m(x, t, e)=m(x,-t,-e)$. The algorithm is not initialised using scattering data; it uses the Jost operator as data, and incorporates it gradually as $|x|$ increases.

The algorithm is derived as follows. An inverse Radon transform of the plasma wave equation (2.2) taking $t, e_{\mathrm{i}}$ into $y$ gives [9]

$$
\left(\Delta_{x}-\Delta_{y}\right) \check{m}(x, y)=\int_{S 2} V(x, e) \check{m}(|x| e, y) \mathrm{d} e .
$$

This in turn may be written as

$$
\left[\left(\frac{\partial^{2}}{\partial x^{2}}+\frac{2}{x} \frac{\partial}{\partial x}\right)-\left(\frac{\partial^{2}}{\partial y^{2}}+\frac{2}{y} \frac{\partial}{\partial y}\right)\right] \check{m}(x, y)=\check{M}(x, y)
$$

where

$$
\check{M}(x, y)=\left(\Delta_{y}^{\mathrm{T}}-\Delta_{x}^{\mathrm{T}}\right) \check{m}(x, y)+\int_{s^{2}} V(x, e) \check{m}(|x| e, y) \mathrm{d} e
$$
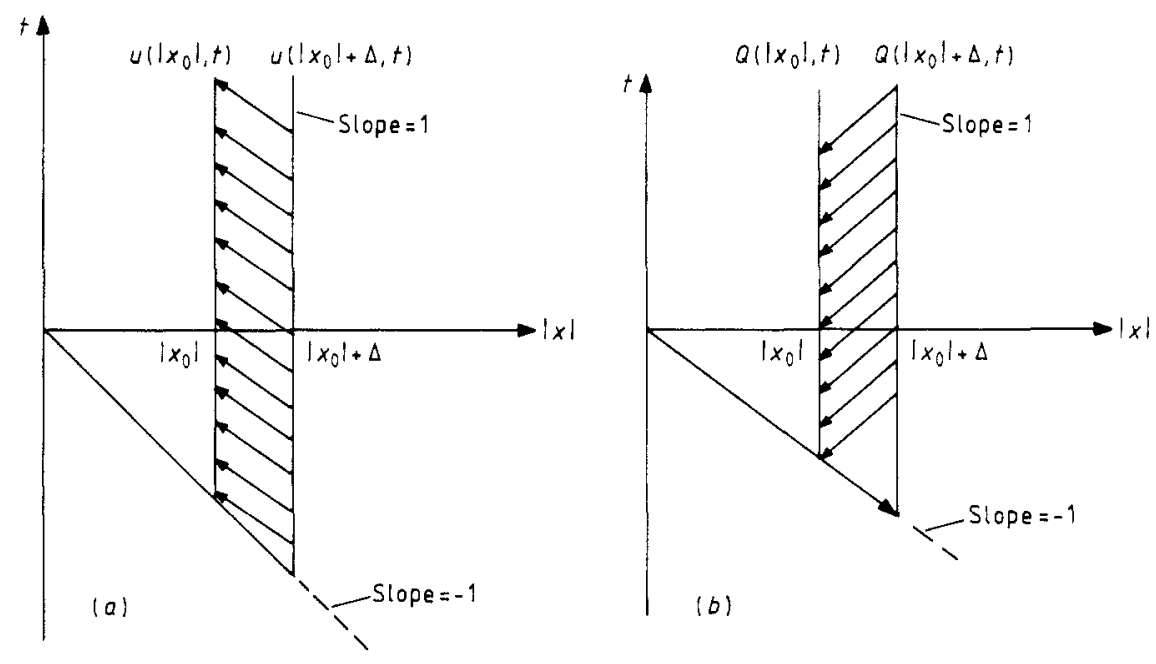

Figure 2. (a) Recursion pattern for updating $u\left(x, t, e_{i}\right)$ in the generalised fast Cholesky algorithm. (b) Recursion pattern for updating $Q\left(x, t, e_{i}\right)$ in the generalised fast Cholesky algorithm. 

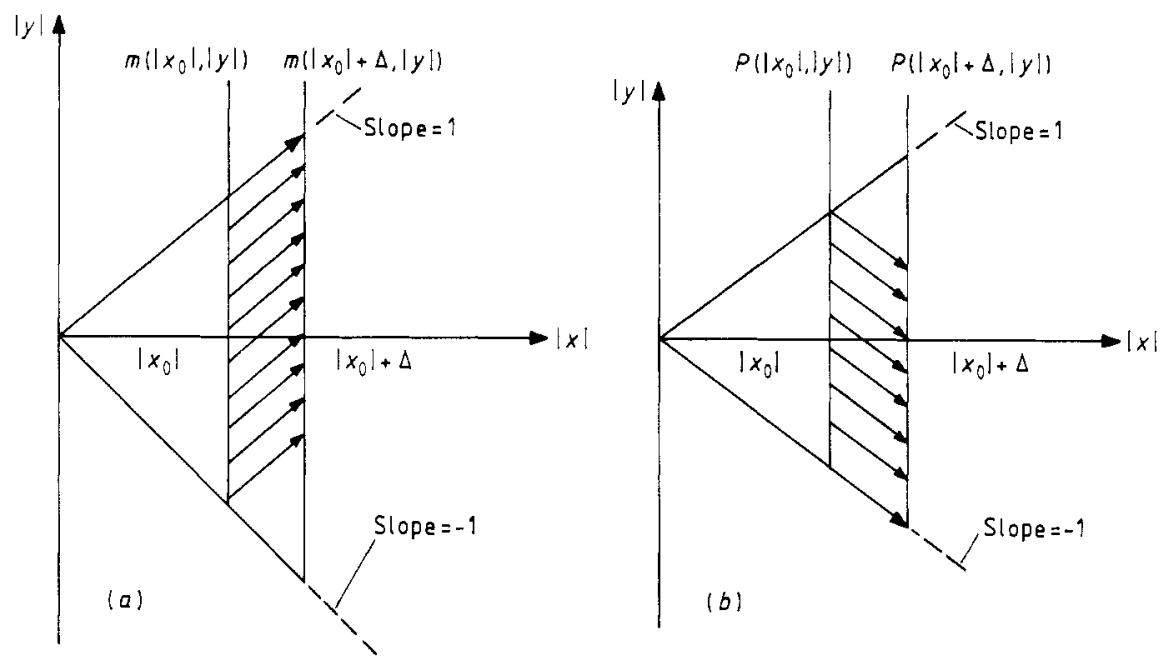

Figure 3. (a) Recursion pattern for updating $m(x, y)$ in the generalised Krein-Levinson algorithm. (b) Recursion pattern for updating $P(x, y)$ in the generalised Krein-Levinson algorithm.

is an auxiliary quantity analogous to $U\left(x, t, e_{i}\right)$. In (4.9) $\Delta_{x}^{\mathrm{T}}$ is the transverse Laplacian (4.2) with respect to $x$ and $\Delta_{y}^{\top}$ is the transverse Laplacian with respect to $y$.

Equation (4.8) may be written as the coupled system of first-order equations

$$
\begin{aligned}
& \left(\frac{\partial}{\partial x}+\frac{\partial}{\partial y}\right)|x||y| \check{m}(x, y)=|x||y| \check{P}(x, y) \\
& \left(\frac{\partial}{\partial x}-\frac{\partial}{\partial y}\right)|x||y| \check{P}(x, y)=|x||y| \check{M}(x, y)
\end{aligned}
$$

where $P(x, y)$ is an auxiliary quantity defined in (4.10a). From (3.19) we also have

$$
V x, e)=-2 \check{P}(x,|x| e) \text {. }
$$

Equations (4.9)-(4.11) can be propagated recursively in increasing $|x|$, for $-|x| \leqslant$ $|y| \leqslant|x|$. The extension to negative values of $|y|$ is made using $y=|y| e_{y}=(-|y|)\left(-e_{y}\right)$; the reason will be given shortly. The algorithm is initialised at the origin $|x|=0$, and proceeds outward in increasing $|x|$ for each $e_{x}=x /|x|$. Suppose the algorithm has proceeded to $\left|x_{0}\right|-\Delta$, so that all quantities are known on the surface of the sphere (i.e. for all $\left.e_{x}\right)$ of this radius, for all $e_{y}$. Then (4.10) are used to compute $\check{m}(x, y)$ and $\check{P}(x, y)$ on the surface of the sphere of radius $\left|x_{0}\right|$, for all $e_{x}, e_{y}$, and $-\left|x_{0}\right| \leqslant|y| \leqslant\left|x_{0}\right|$. Next, (4.11) is used to compute $V(x, e)$ on the surface of this sphere, for all $e$. Finally, (4.9) is used to compute $\grave{M}(x, y)$ on the surface of this sphere. At this point all quantities are known on the surface of the sphere of radius $\left|x_{0}\right|$ and the recursion is complete.

The recursion patterns for this algorithm are illustrated in figure 3 . Note that by causality all quantities are zero for $|y|>|x|$, so no information is lost in the unshaded regions. Note also that since the region of support $-|x| \leqslant|y| \leqslant|x|$ is growing steadily larger, there are two holes in the recursions at $\check{m}(x,-|x|)$ and $\breve{P}(x,|x|)$. The second 
hole is precisely the value needed to compute $V(x, e)$. These holes are filled as follows. First, we have

$$
\check{m}\left(x,-|x|, e_{y}\right)=\check{m}\left(x,|x|,-e_{y}\right),
$$

which is the reason for making the extension of $|y|$ to negative values. The other hole is filled by going back to the integral equation (3.8). Setting $y=|x| e_{y}$ in (3.8) gives

$$
\check{m}\left(x,|x| e_{y}\right)=\check{H}\left(x,|x| e_{y}\right)-\int_{|z|<|x|} \check{m}(x, z) \check{H}\left(z,|x| e_{y}\right) \mathrm{d} z
$$

where $\breve{H}(x, y)$ is defined in (3.9) as the double inverse Fourier transform of $\left(\left(J^{\mathrm{H} J} J\right)^{-1}-I\right)\left(k,-e_{1}, e_{2}\right)$. Applying $(4.10 a)$ to (4.13) gives a messy expression for $\check{P}(x,|x|)$. Note that the scattering data, in the form of the Jost operator or spectral function, are used only for the computation of $P(x,|x|)$.

This algorithm is termed a generalised Krein-Levinson algorithm since it resembles the one-dimensional Krein-Levinson algorithm [21] in the following ways. First, the quantity being propagated is the regular solution, which is causal and has compact but growing support. Second, the scattering parameter (potential or reflection coefficient) is not computed automatically, as with the previous algorithm, but must be computed using the inner product expression (4.13). Finally, the scattering data are used only in the inner product expression, and thus must be stored.

\subsection{Comparison of the algorithms}

The two algorithms, like the generalised Marchenko and Gel'fand-Levitan integral equations they solve, look similar but are in fact quite different. Apart from the differences in support and domain of operation (Radon against inverse Radon), the generalised fast Cholesky algorithm is computationally preferable for three reasons. First, it is not necessary to solve the integral equation (3.12) for the Jost operator; near-field scattering data are used directly. Second, the scattering data are used to initialise the algorithm; they may then be discarded, saving storage. Finally, avoiding the inner product expression (4.13) saves a considerable amount of computation. All of these advantages also apply in the one-dimensional case [21].

The computational advantage of these algorithms over the integral equations is considerable. Solution of the integral equations by discretising each coordinate axis (including time) into $\mathrm{O}(N)$ points amounts to inverting an $N^{3} \times N^{3}$ matrix $N^{3}$ times. If gaussian elimination is used this requires $\mathrm{O}\left(N^{12}\right)$ computations! The two fast algorithms only require $N$ updates requiring $\mathrm{O}\left(N^{7}\right)$ computations each, for a total of $\mathrm{O}\left(N^{8}\right)$ computations-a considerable saving. Note that the logarithm of the number of computations is reduced by one-third from $12 \log N$ to $8 \log N$, matching the reduction from $3 \log N$ to $2 \log N$ for the one-dimensional case. The generalised fast Cholesky algorithm can also be parallelised almost completely; this may make the algorithm computationally feasible on a parallel machine, although no numerical results are available as yet.

\section{Application to estimation of random fields}

We now apply the results of this paper to the problem of computing the linear leastsquares estimate of a three-dimensional random field. Following [19], the problem is 
formulated as an inverse scattering problem with a non-local potential, which can then be solved using the fast algorithms of $\S 4$.

The estimation problem considered is a filtering problem of computing the linear least-squares estimate of a zero-mean, real-valued random field $z(x)$ on the surface of a sphere of radius $|x|$, from noisy observations

$$
w(x)=z(x)+v(x)
$$

inside the sphere. $v(x)$ is a zero-mean, real-valued, white-noise field with covariance

$$
E[v(x) v(y)]=\delta(x-y)
$$

while $z(x)$ is uncorrelated with $v(x)$ and has covariance function

$$
E[z(x) z(y)]=k(x, y)
$$

where the function $k(x, y)$ is positive definite and has the generalised displacement property [19]:

$$
\left(\Delta_{x}-\Delta_{y}\right) k(x, y)=0 \text {. }
$$

The structure of $k(x, y)$ implied by $(5.4)$ reduces the number of degrees of freedom in the function $k(x, y)$ from six to five. This is still a far more general case than the case of a homogeneous random field having covariance $k(x-y)$ (three degrees of freedom) treated in [20], or the case of an isotropic random field having covariance $k(|x-y|)$ (one degree of freedom) treated in [22]. Note that both homogeneous and isotropic random fields are included as special cases of the property (5.4).

The estimate $\hat{z}(x)$ of $z(x)$ has the form

$$
\hat{z}(x)=\int_{|y|<|x|} h(x, y) w(y) \mathrm{d} y .
$$

By the orthogonality principle the filter $h(x, y)$ is determined by the three-dimensional Wiener-Hopf integral equation

$$
k(x, y)=h(x, y)+\int_{z|<| x i} h(x, z) k(z, y) \mathrm{d} z \quad|y|<|x| .
$$

Without loss of generality, we define $h(x, y)=0$ for $|y|>|x|$.

Applying the operator $\left(\Delta_{x}-\Delta_{y}\right)$ to the integral equation (5.6) and using the generalised displacement property (5.4), Green's theorem, and the unicity of solution to (5.6) when $k(x, y)$ is positive definite yields, after some algebra (see [20]),

$$
\left(\Delta_{x}-\Delta_{y}\right) h(x, y)=\int_{S^{2}} V(x, e) h(|x| e, y) \mathrm{d} e
$$

where the non-local filter potential $V(x, e)$ is defined as

$$
V(x, e)=-\frac{2}{|x|^{2}} \frac{\mathrm{d}}{\mathrm{d}|x|}|x|^{2} h(x,|x| e) .
$$

Then a Radon transform of (5.7) taking $y$ into $t$ and $e_{i}$ yields

$$
\left(\Delta-\partial^{2} / \partial t^{2}\right) \hat{h}\left(x, t, e_{\mathrm{i}}\right)=\int_{s=} V(x, e) \hat{h}\left(|x| e, t, e_{\mathrm{i}}\right) \mathrm{d} e
$$


and clearly the filtering problem has been formulated as an inverse scattering problem with a non-local potential. In particular, the optimal filter $h(x, y)$ is the inverse Radon transform of the regular solution. Note that (5.8) is identical to (3.19).

It is not surprising that the potential is non-local. Since $k(x, y)$ has five degrees of freedom, the set of potentials that characterise it must also have five degrees of freedom. Note that non-local potentials do not arise in the isotropic case considered in [22], due to symmetry. For the homogeneous case considered in [20], this symmetry no longer holds, and the potential is non-local.

The structure (5.4) of $k(x, y)$ implies that its double Fourier transform is zero except for its on-shell values. More specifically,

$$
\mathscr{F}_{x \rightarrow k_{1} e_{1}} \mathscr{F}_{y \rightarrow k_{2} c_{2}}(\delta(x-y)+k(x, y))=M\left(k, e_{1}, e_{2}\right) \delta\left(\left|k_{1}\right|^{2}-\left|k_{2}\right|^{2}\right)
$$

for some function $M\left(k, e_{1}, e_{2}\right)$. Since $\delta(x-y)+k(x, y)$ is positive definite, the function $M\left(k, e_{1}, e_{2}\right)$ can be factorised into

$$
M\left(k, e_{1}, e_{2}\right)=\int_{S^{2}} J^{-1}\left(k, e_{1}, e_{3}\right) J^{-1}\left(k, e_{2}, e_{3}\right)^{*} \mathrm{~d} e_{3}
$$

where $J^{-1}\left(k, e_{1}, e_{2}\right)$ is analytic in $k$ in the lower half-plane. It is evident that $J^{-1}\left(k, e_{1}\right.$, $e_{2}$ ) can be interpreted as the kernel of an inverse Jost operator, which can be used in the generalised Krein-Levinson algorithm.

\section{Conclusion}

The Schrödinger equation inverse scattering problem with a non-local potential operator diagonal in the radius has been solved using generalised Marchenko and Gel'fand-Levitan integral equations. This extends the work of Newton [1-3] to the case of the non-local potentials, and generalises the relation between the onedimensional versions of these equations [16] to the three-dimensional case, for a nonlocal potential. Although integral equations for this problem were given in [9-11], this presentation clarifies the relationship between the integral equations and generalises one-dimensional results, unlike [9-11]. In particular, both integral equations were shown to be interpretable as performing Gram-Schmidt orthogonalisations of freespace wavefunctions.

The major contribution of the paper was the development of generalised Krein-Levinson and fast Cholesky algorithms for solving this problem. Again the relationship between these algorithms was noted; the fast Cholesky algorithm, as in the one-dimensional case, was noted to be preferable computationally. The parallelisability of this algorithm may well make it computationally practical; this is a subject of current research.

There were three reasons for considering a non-local potential operator diagonal in the radius. The first was that such a potential can be recovered from the scattering amplitude with little more trouble than for a local potential, and without encountering the characterisation problem associated with a local potential, which manifests itself as the miracle of Newton [1]. The second was that although this problem is noncausal, the scattered field is still zero for $t<-|x|$; this makes one of the fast algorithms possible. The third was that this problem is of some interest, as shown by its application to the problem of estimating a random field in $\$ 5$. 


\section{Acknowledgments}

The author wishes to thank Dr Margaret Cheney for bringing reference [10] to his attention, and the referees for bringing references [11-14] and [18] to his attention.

\section{References}

[1] Newton R G 1980 Inverse scattering II. Three dimensions J. Math. Phys. 21 1698-715

[2] Newton R G 1981 Inverse scattering III. Three dimensions, continued J. Math. Phys. 22 2191-200

[3] Newton R G 1982 Inverse scattering IV. Three dimensions: generalised Marchenko construction with bound states, and generalized Gel'fand-Levitan equations J. Math. Phys. 23 594-604

[4] Rose J H, Cheney M and DeFacio B 1984 The connection between time and frequency domain threedimensional inverse scattering methods $J$. Math. Phys. 25 2995-3000

[5] Rose J H, Cheney M and DeFacio B 1985 Three-dimensional inverse scattering: plasma and variablevelocity wave equations $J$. Math. Phys. 26 2803-13

[6] Rose J H, Cheney M and DeFacio B 1986 Determination of the wave field from scattering data Phys. Rev. Lett. $57783-6$

[7] Yagle A E 1986 Differential and integral methods for multi-dimensional inverse scattering problems J. Math. Phys. 27 2584-91

[8] Yagle A E 1987 Multi-dimensional inverse scattering: an orthogonalization formulation J. Math. Phys. 28 1481-91

[9] Kay I and Moses H E 1961 A simple verification of the Gelfand-Levitan equation for the threedimensional scattering problem Commun. Pure Appl. Math. 14 435-45

[10] Kay I and Moses H E 1961 The determination of the scattering potential from the spectral measure function. V. The Gel'fand-Levitan equation for the three dimensional scattering problem Nuovo Cimento 22 689-705

[11] Moses H E 1980) Exact solutions for the three-dimensional Schrödinger equation with quasi-local potentials obtained from a three-dimensional Gel'fand-Levitan equation. Examples of totally reflectionless scattering Mathematical Methods and Applications of Scattering Theory ed J A DeSanto et al (New York: Springer)

[12] Abraham P B, DeFacio B and Moses H E 1983 Parity-dependent potentials for the one-dimensional Schrödinger equation obtained from inverse spectral theory J. Phys. A: Math. Gen. Phys. 1630316

[13] Moses H E 1956 Calculation of the scattering potential from reflection coefficients Phys. Rev. $102559-$ 67

[14] Moses H E 1979 Jost solutions and Green's functions for the three-dimensional Schrödinger equation J. Math. Phys. $201151-6$

[15] Yagle A E and Levy B C 1985 A fast algorithm solution of the inverse problem for a layered acoustic medium probed by spherical harmonic waves $J$. Acoust Soc. Am. 78 729-37

[16] Burridge R 1980 The Gelfand-Levitan, the Marchenko, and the Gopinath-Sondhi integral equations of inverse scattering theory, regarded in the context of inverse impulse-response problems Wave Motion 2 305-23

[17] DeFacio B and Rose J H 1985 Inverse scattering theory for the non-spherically symmetric threedimensional plasma wave equation Phys. Rev. A 31 897-902

[18] Moses H E 1980 A kernel of Gel fand-Levitan type for the three-dimensional Schrödinger equation $J$. Math. Phys. $2183-9$

[19] Yagle A E 1987 Connections between three-dimensional inverse scattering and the linear least-squares estimation of random fields Acta Appl. Math. to be published

[20] Yagle A E 1987 A fast algorithm for linear estimation of three-dimensional homogeneous anisotropic random fields Proc. IEEE Int. Conf. on Acoustics, Speech and Signal Processing, Dallas pp $1712-5$

[21] Bruckstein A M, Levy B C and Kailath T 1985 Differential methods in inverse scattering SIAMJ. Appl. Math. 45 312-35

[22] Levy B C and Tsitsiklis J N 1985 A fast algorithm for linear estimation of two-dimensional isotropic random fields IEEE Trans. Info. Th. IT-31 635-44 